\title{
Frustration and Transition Point of Ising Spin Glasses
}

\author{
Ryoji MIYAZAKI* \\ Department of Physics, Tokyo Institute of Technology, Meguro, Tokyo 152-8551, Japan
}

Received April 1, 2013; final version accepted May 31, 2013

\begin{abstract}
A relationship between frustration and the transition point at zero temperature of the Ising spin glasses is reported. We find that the concentration of antiferromagnetic bonds in the system is in good agreement with the critical concentration at zero temperature when the derivative of the average number of frustrated plaquettes with respect to the average number of antiferromagnetic bonds is equal to unity. This relation is confirmed in the Ising spin glasses with binary couplings on the two-dimensional lattices, the hierarchical lattices, and the three-body Ising spin glasses with binary couplings on the two-dimensional lattices. The same argument in the SherringtonKirkpatrick model yields a point that is identical to the replica-symmetric solution of the transition point at zero temperature.
\end{abstract}

KEYWORDS: spin glasses, quantum error-correcting codes, frustration, phase transitions, ground states

\section{Introduction}

Spin-glass theory has a deep connection with information processing [1]. Indeed, an order-disorder phase boundary in the finite-dimensional spin-glass model corresponds to the accuracy thresholds of topological quantum errorcorrecting codes [2]. It is natural to expect that we can extract the accuracy thresholds from the knowledge of spin glasses based on the investigation of the latter over decades. Nevertheless, the phase diagrams in the finite-dimensional spin glasses have not been clarified. One of the strategies for derivation of the accuracy thresholds is to develop the finite-dimensional spin-glass theory.

Concerning the phase diagram in spin glasses, there has been a proposition based on the property of the Nishimori line $[1,3]$, which is a special line across the phase diagram as depicted in Fig. 1, that the phase transition between the ferromagnetic and non-ferromagnetic phases at lower temperatures than the Nishimori line is induced by a geometric nature $[1,4]$. The entropy of frustration distribution has a singularity at the transition point on the Nishimori line. Frustration is a geometric quantity defined later, which corresponds to Ising vortices or defects where a check operator takes the value -1 in terms of topological quantum error-correcting codes [2]. The singularity is guessed to induce the phase transition also at lower temperatures than the Nishimori line.

To illustrate the concept of frustration, we consider the $\pm J$ Ising model on the square lattice governed by the Hamiltonian,

$$
H=-\sum_{\langle i j\rangle} J_{i j} S_{i} S_{j}
$$

where $J_{i j}$ takes either $J(>0)$ with probability $1-p$ or $-J$ with probability $p$, and $S_{i}$ is an Ising variable $\left(S_{i}= \pm 1\right)$. The summation runs over all the nearest neighbors. Negative bonds correspond to errors in error-correcting codes. If the number of negative (antiferromagnetic) coupling constants is odd in a loop composed of bonds, there is no spin configuration permitting all bonds to be in the lower-energy state $\left(-J_{i j} S_{i} S_{j}=-J\right)$. The product of coupling constants $\prod_{\langle i j\rangle \in c} J_{i j}$ over an arbitrary loop $c$ is called frustration [1,5]. If frustration of a loop has a negative value, the loop is regarded as a "frustrated loop." The frustration of minimal loops, namely plaquettes, are mainly investigated. The list of all locations of frustrated plaquettes in the system provides the distribution of frustration, that is, a syndrome in topological quantum error-correcting codes. The entropy of frustration distribution is defined as

$$
S=-\sum_{x} P(x) \log P(x)
$$

where $P(x)$ denotes the probability of the distribution $x$ of frustration.

This work is supported by the Research Fellowship of the Japan Society for the Promotion of Science.

*Corresponding author. E-mail: miyazaki@stat.phys.titech.ac.jp 


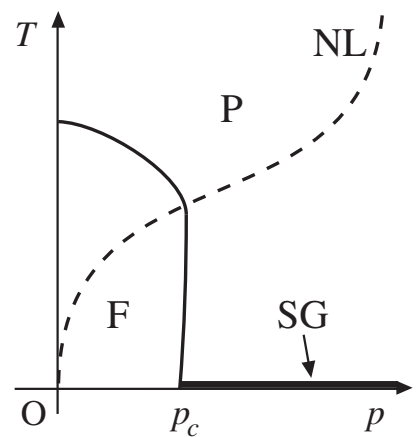

Fig. 1. The phase diagram of the $\pm J$ Ising model in two dimensions. The vertical and horizontal axes express temperature and the concentration of antiferromagnetic bonds, respectively. The symbols F, P, and SG denote the ferromagnetic, paramagnetic, and spin-glass phases, respectively. The boundary of ferromagnetic phase corresponds to the accuracy threshold of topological quantum error-correcting codes [2]. The Nishimori line (NL) is drawn as a dashed line. The critical concentration at zero temperature is denoted by $p_{c}$

The geometry-induced phase transition has not been confirmed, and the relationship between frustration and the phase transitions in spin glasses is not clear. Thus, we try to detect phase transitions only by considering frustration in the present paper. We particularly observe the change of the average number of frustrated plaquettes as the average number of antiferromagnetic bonds is increased. Note that plaquette means an elementary loop composed of bonds in the lattice, which cannot be divided into any other loops. We find that when the increment of the average number of frustrated plaquettes is equal to that of antiferromagnetic bonds, the concentration of antiferromagnetic bonds in the system is in good agreement with the critical concentration at zero temperature estimated by other approaches [6-11].

The present paper is organized as follows. In $\S 2$, we consider the $\pm J$ Ising model [Eq. (1.1)] on the two-dimensional lattices and the hierarchical lattices. An interesting relationship between the average number of frustrated plaquettes and the transition point at zero temperature is found in this section. We confirm the same relationship in the model with the three-body interactions in §3. The Sherrington-Kirkpatrick (SK) model [12] is investigated in $\S 4$. The final section is devoted to summary and discussion.

\section{Ising Spin Glasses with Binary Couplings}

Let us consider frustration in the $\pm J$ Ising model [Eq. (1.1)] on the square lattice. We investigate the frustration of plaquettes, which are the squares composed of four bonds in this case. We calculate the configurational average over the distribution of $\left\{J_{i j}\right\}$ of the number $N_{f}^{\mathrm{sq}}$ of frustrated plaquettes $[13,14]$. Note that the periodic boundary condition is assumed. In other words, the system lies on a torus. The concentration of antiferromagnetic bonds, or errors, is denoted by $p$. We have

$$
\begin{aligned}
N_{f}^{\mathrm{sq}}(p):=\left[N_{f}^{\mathrm{sq}}\right]_{p} & =\left[\sum_{\mathrm{c}} \frac{1}{2}\left(1-\prod_{\langle i j\rangle \in c} \frac{J_{i j}}{J}\right)\right]_{p} \\
& =\frac{N}{2}\left\{1-(1-2 p)^{4}\right\},
\end{aligned}
$$

where $N$ is the number of spins, and $[\cdots]_{p}$ denotes the configurational average with $p$. The number of plaquettes is equal to the number of spins on the square lattice. The summation runs over all plaquettes $c$.

To understand the creation of frustration in the system, we depict Fig. 2. Dashed lines express the square lattice and a circle denotes a frustrated plaquette, which is a end of a path through antiferromagnetic bonds. When $p \simeq 0$ [Fig. 2(a)], there are few antiferromagnetic bonds on the lattice, so an additional antiferromagnetic bond certainly makes two ends, that is, two frustrated plaquettes. As the number of segments increases, the system is crowded, and it becomes difficult to make two ends [Fig. 2(b)]. To explicitly calculate the change of the number of frustrated plaquettes, we differentiate $N_{f}^{\mathrm{sq}}(p)$ with respect to the average number $N_{a}^{\mathrm{sq}}(p)$ of antiferromagnetic bonds, which is equal to $2 N p$ in the case of the square lattice. The resulting derivative is

$$
v^{\mathrm{sq}}(p):=\left(\frac{d N_{a}^{\mathrm{sq}}(p)}{d p}\right)^{-1} \frac{d N_{f}^{\mathrm{sq}}(p)}{d p}=2(1-2 p)^{3} .
$$

This quantity $v^{\mathrm{sq}}(p)$ expresses the ratio of the increment of the number of frustrated plaquettes to that of antiferromagnetic bonds.

When $p \simeq 0$, the increment of the number of frustrated plaquettes is larger than that of antiferromagnetic bonds. When $p \simeq 1 / 2$, the reverse is realized. There is a turning point where the increment of the number of frustrated plaquettes is equal to that of antiferromagnetic bonds. It is identified with the point $v^{\mathrm{sq}}\left(p^{\mathrm{sq}}\right)=1$, where 
(a)

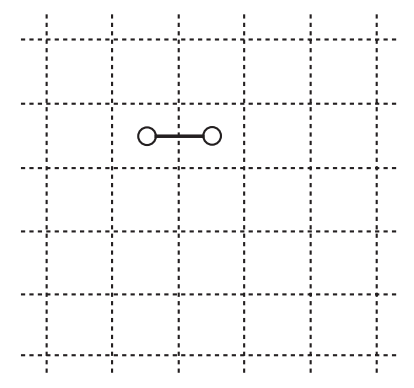

(b)

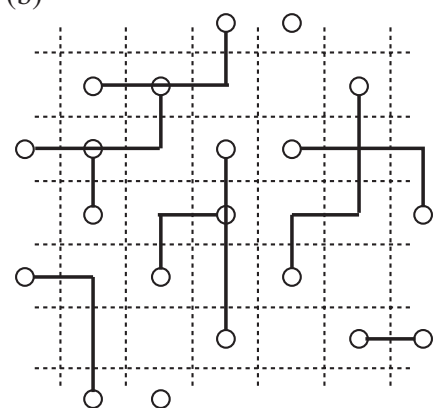

Fig. 2. Antiferromagnetic bonds and frustrated plaquettes on the square lattice. Thick segments traverse antiferromagnetic bonds, and circles denote frustrated plaquettes. Two cases (a) $p \simeq 0$ and (b) $p \simeq 1 / 2$ are shown.

Table 1. The equations and solutions of $v(p)=1$ and the locations $p_{c}$ of the transition points at zero temperature in several two-dimensional lattices.

\begin{tabular}{lccl}
\hline Lattice & Our equation & Our solution & \multicolumn{1}{c}{$p_{c}$} \\
\hline Square & $2\left(1-2 p^{\mathrm{sq}}\right)^{3}=1$ & 0.1031 & $0.103[6]$ \\
& & & $0.1045(11)[7]$ \\
& & $0.103(1)[8]$ \\
& & & $0.1031(1)[9]$ \\
Triangle & $2\left(1-2 p^{\mathrm{tri}}\right)^{2}=1$ & 0.1464 & $0.159[6]$ \\
& & & $0.166(3)[10]$ \\
Hexagon & $2\left(1-2 p^{\mathrm{hex}}\right)^{5}=1$ & 0.0647 & $0.065[6]$ \\
& & & $0.0667[10]$ \\
Kagomé & $\left(1-2 p^{\mathrm{kag}}\right)^{2}+\left(1-2 p^{\mathrm{kag}}\right)^{5}=1$ & 0.0956 & $0.095[6]$ \\
Dual of kagomé & $2\left(1-2 p^{\mathrm{d}-\mathrm{kag}}\right)^{3}=1$ & 0.1031 & $0.116[6]$ \\
Extended kagomé & $\frac{2}{3}\left(1-2 p^{\mathrm{ex}-\mathrm{kag}}\right)^{2}+\frac{4}{3}\left(1-2 p^{\mathrm{ex}-\mathrm{kag}}\right)^{11}=1$ & 0.0468 & $0.041[6]$ \\
Dual of extended kagomé & $2\left(1-2 p^{\mathrm{d}-\mathrm{ex}-\mathrm{kag}}\right)^{2}=1$ & 0.1464 & $0.205[6]$ \\
\hline
\end{tabular}

(a)

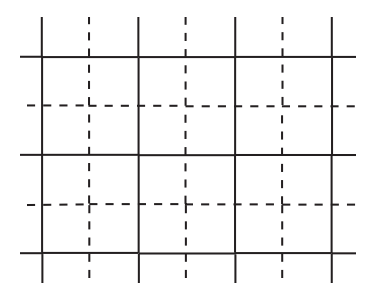

(b)

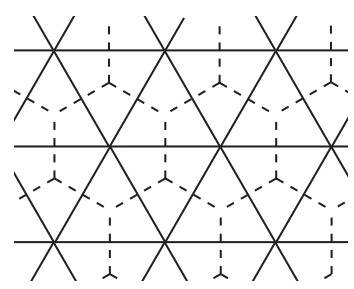

(c)

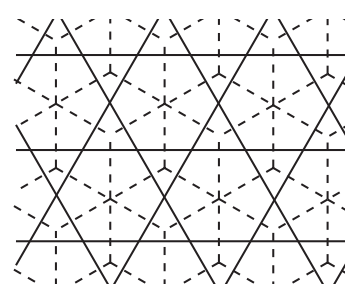

(d)

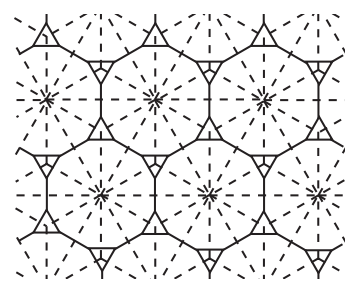

Fig. 3. Two dimensional lattices listed in Table 1. Solid lines express (a) the square, (b) triangle, (c) kagomé, and (d) extended kagomé lattices. The dual of these lattices are drawn as dashed lines.

$$
p^{\mathrm{sq}}=\frac{1}{2}-\left(\frac{1}{2}\right)^{4 / 3} \simeq 0.1031 .
$$

Surprisingly, this value is very close to the phase-transition point at zero temperature estimated by other numerical approaches (Table 1). This agreement suggests that the increment of the number of frustrated plaquettes might control the phases where the system lies. Since temperature has not been considered, it is reasonable that the temperature at which our value indicates the transition point is zero. Although our argument yields an extraordinarily accurate correspondence, we cannot regard it as the derivation of the phase-transition point since we have not found any singularity in physical quantities.

The results of other two-dimensional lattices (Fig. 3) are listed in Table 1. The solutions of $v(p)=1$ in several lattices show fairly good agreement as the case of the square lattice. These results demonstrate that the correspondence between the transition point and the solution of $v(p)=1$ is not accidental on the square lattice. In addition, our results in the dual of kagomé and extended kagomé lattices, which consist of squares and triangles, respectively, suggest that our argument imperfectly reflects the global structure of lattices but produces an effective approximation with the local structure of lattices. 


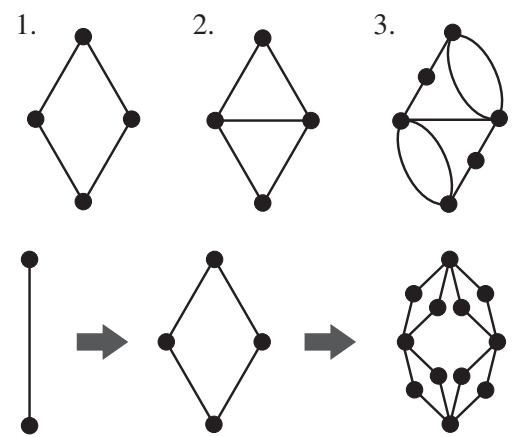

Fig. 4. The units of hierarchical lattices 1,2, and 3 examined in the present paper (top) and the replacement of each single bond with the unit of the lattice 1 (bottom). Dots represent sites for spins.

Table 2. The equations and solutions of $v(p)=1$ and the locations $p_{c}$ of the transition points at zero temperature in the hierarchical lattices in Fig. 4. The indices $k$ of summations in the equations run from 1 to infinity.

\begin{tabular}{|c|c|c|c|}
\hline Lattice & Our equation & Our solution & $p_{c}$ \\
\hline 1 & $\sum 2^{-k+1}\left(1-2 p^{\text {hier }-1}\right)^{2^{k+1}-1}=1$ & 0.0523 & $0.0785(3)$ \\
\hline 2 & $\sum 3\left(\frac{2}{5}\right)^{k}(1-2 p)^{3 \cdot 2^{k-1}-1}=1$ & 0.0911 & $0.1065(1)$ \\
\hline 3 & $\sum 3^{-k-1}\left\{4\left(1-2 p^{\text {hier }-3}\right)^{2 \cdot 3^{k-1}-1}+8\left(1-2 p^{\text {hier }-3}\right)^{4 \cdot 3^{k-1}-1}\right\}=1$ & 0.0813 & $0.1049(3)$ \\
\hline
\end{tabular}

We next consider the hierarchical lattices [15], which are generated by iterating the process that each single bond is replaced with a unit of the lattice as depicted in Fig. 4. Since we can obtain the accurate transition point on the hierarchical lattices with the renormalization-group calculations $[15,16]$, the lattices are a good testing ground for the comparison of estimates of the transition point. We examine the lattices 1, 2, and 3 in Fig. 4.

In the case of the lattice 1, the derivative corresponding to Eq. (2.2) in the infinite-volume limit is

$$
v^{\text {hier-1 }}(p)=\lim _{n \rightarrow \infty} \sum_{k=1}^{n} 2^{-k+1}(1-2 p)^{2^{k+1}-1} .
$$

Since it is difficult to solve the equation $v^{\text {hier-1 }}\left(p^{\text {hier-1 }}\right)=1$, we numerically evaluate the solution in the case of $n=20$. We then obtain the value $p^{\text {hier-1 }}=0.0523$. The same value is obtained even in the case of $n=30$. Thus, it is reasonable to interpret this value as the actual solution.

To compare this value with the location of the transition point at zero temperature, we derive the transition point with another reliable approach, namely the renormalization-group method proposed by Nobre [16]. The size of the system is set equal to $10^{6}$. After the renormalization-group transformations 30 times, which is the reverse process of the construction of the hierarchical lattice, if the average of magnitudes of resulting couplings is larger (smaller) than 10 $\left(10^{-3}\right)$, we regard the system to be in the ferromagnetic (paramagnetic or spin-glass) phase. To reduce statistical errors, we run 100 samples.

The solutions of $v(p)=1$ have a bit difference but qualitatively close to the estimates of the transition points at zero temperature in the respective lattices listed in Table 2. It is remarkable that the results correctly express the order of locations of the transition points. In particular, a slight difference in the lattice 2 and 3 is distinguished. This result demonstrates the effectiveness of our argument to qualitatively predict the transition points.

\section{Three-Body Ising Spin Glasses with Binary Couplings}

In this section we apply our method to a different type of models from previous ones, the $\pm J$ Ising model with the three-body interactions,

$$
H=-\sum_{\langle i, j, k\rangle} J_{i j k} S_{i} S_{j} S_{k} .
$$

Couplings $J_{i j k}$ are governed by the same distribution as the previous models with the two-body interactions. One of the reasons to investigate this model is that the order-disorder phase boundary in this model is equivalent to the accuracy threshold in a kind of topological quantum error-correcting codes, namely color codes [17, 18].

To illustrate frustration in this model, let us consider the model on the triangular lattice. Three spins on the same triangle interact with each other, and couplings $J_{i j k}$ reside on triangles. A loop in this model is composed of triangles. We call the elementary loop which can be frustrated "unit of frustration," which corresponds to a plaquette in the previous cases. The unit of frustration in the triangular lattice is the minimal hexagon composed of six triangles. If the number of negative couplings in a unit of frustration is odd, the unit is frustrated. 
Table 3. The equations and solutions of $v(p)=1$ and the critical concentrations $p_{c}$ and $p_{m}$ of negative triangles at zero temperature and on the Nishimori line, respectively, in the three-body $\pm J$ model on the triangular and Union-Jack lattices. The Union-Jack lattice is depicted in Fig. 5. The value $p_{c}$ is expected to be sightly smaller than the value $p_{m}$ in the triangular lattice as in the Union-Jack lattice.

\begin{tabular}{lcccc}
\hline Lattice & Our equation & Our solution & $p_{c}$ & \multicolumn{1}{c}{$p_{m}$} \\
\hline Triangle & $3\left(1-2 p^{3, \text { tri }}\right)^{5}=1$ & 0.0986 & & $0.1097(1)[19]$ \\
& & & & $0.109(2)[18]$ \\
Union-Jack & $\left(1-2 p^{3, \mathrm{U}-\mathrm{J}}\right)^{3}+2\left(1-2 p^{3, \mathrm{U}-\mathrm{J}}\right)^{7}=1$ & 0.0941 & $0.1056(1)[11]$ & $0.10925(5)[19]$ \\
& & & & $0.109(2)[20]$ \\
\hline
\end{tabular}

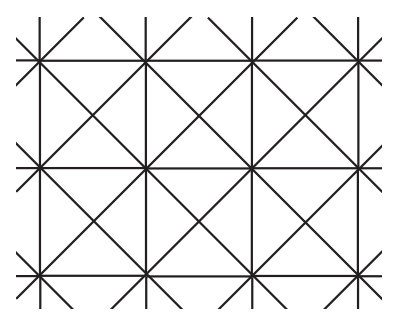

Fig. 5. The Union-Jack lattice.

The derivative $v$ in this case is represented as

$$
v^{3, \text { tri }}(p)=3(1-2 p)^{5}
$$

We obtain the value $p^{3, \text { tri }}=0.0986$, where $v^{3, \text { tri }}\left(p^{3, \text { tri }}\right)=1$. Since the transition point at zero temperature in the model, to the best of our knowledge, has not been numerically estimated in any other studies, we compare this value with the concentration of negative triangles at the transition point on the Nishimori line instead of the point at zero temperature. The critical concentrations on the Nishimori line and at zero temperature are expected to have little difference. Indeed, the two values are very close in the case of the Union-Jack lattice (Fig. 5), where the transition point at zero temperature has been estimated [11]. The values are compared in Table 3. The result shows fairly good agreement. It is noted that our value from $v(p)=1$ in the triangular lattice is a little larger than that in the Union-Jack lattice. This fact corresponds to a tiny difference between the critical concentrations on the Nishimori line in the two lattices which has been derived by the preceding study [19] accepted as a highly accurate prediction. Furthermore, comparing the result for the triangular lattice with the result for the models with the two-body interactions on the same lattice shown in Table 1, we find that our argument properly distinguishes the number of bodies in the couplings.

\section{SK Model}

We investigate the standard infinite-range model in spin glasses called the SK model [12],

$$
H=-\sum_{i<j} J_{i j} S_{i} S_{j}
$$

where any two spins interact with each other. The couplings $J_{i j}$ are governed by the Gaussian distribution,

$$
P\left(J_{i j}\right)=\sqrt{\frac{N}{2 \pi}} \exp \left(-\frac{N}{2}\left(J_{i j}-\frac{J_{0}}{N}\right)^{2}\right),
$$

where $N$ denotes the number of spins. This probability distribution has the following features, which the binary distribution in the previous sections does not have. A random continuous variable $J_{i j}$ includes zero. The mean value can be finite.

We consider the number of frustrated plaquettes. A plaquette in this model is a triangle composed of three bonds. Since quenched variables $J_{i j}$ can take arbitrary real values, their product over a plaquette also takes arbitrary values. However, we do not take account of the magnitude of the product, since our attention is focused on whether or not a plaquette is frustrated. This is a generalization of the method developed in the previous sections.

In the infinite-volume limit $N \rightarrow \infty$ the derivative corresponding to Eq. (2.2) is

$$
v^{\mathrm{SK}}\left(J_{0}\right)=\frac{2}{\pi} J_{0}^{2} .
$$

The solution of the equation $v^{\mathrm{SK}}\left(J_{0}^{\mathrm{SK}}\right)=1$ is $J_{0}^{\mathrm{SK}}=\sqrt{\pi / 2}$. This result does not agree with the well known exact 
transition point at zero temperature $J_{0}=1$ [21] but precisely identical with the transition point under the assumption of replica symmetry [12], which is derived from the equation of state of the ferromagnetic order parameter $m$ at zero temperature,

$$
m=\operatorname{erf}\left(\frac{J_{0}}{\sqrt{2}} m\right)
$$

This equation has a solution with $m \neq 0$ if the slope of the function of the right-hand side at $m=0$, which is $\sqrt{2 / \pi} J_{0}$, is larger than 1 . Therefore, the phase-transition point is determined by $v^{\mathrm{SK}}\left(J_{0}\right)=1$ that is identical to the condition in our argument. Our result with frustration accords with the replica-symmetric solution not only in the solution but also in the equation for the determination of the transition point.

\section{Summary and Discussion}

We have reported an interesting relation between frustration and the transition point of the Ising spin glasses. The relation reveals that the concentration of antiferromagnetic bonds in the system is rather close to the critical concentration at zero temperature when the derivative of the average number of frustrated plaquettes with respect to the average number of antiferromagnetic bonds is equal to unity. This relation is confirmed in kinds of models. In particular, the cases in the two-dimensional lattices show good correspondence. In the SK model the solution derived from our argument exactly agrees with the transition point under the assumption of replica symmetry, and the slope of the average number of frustrated plaquettes corresponds to the slope of the function in the equation of state of the ferromagnetic order parameter at zero temperature. This relation is mapped onto the relation between the Ising vortices or defects and the accuracy thresholds with the chain of the shortest length of topological quantum error-correcting codes [2].

Although we have detected no conventional sign of phase transitions, the agreements between our values and the transition points are naturally regarded not to be accidents. One of the reasons is that our argument attains pretty accurate predictions in most of the models which we have applied the method to. The variety of models which the agreements are found is especially remarkable. Thus, our argument is expected to yield approximate locations of the transition points.

Simpleness of our calculation in contrast with the accuracy has practical utility in the context of topological quantum error-correcting code. Our method is useful for the first approximation of the accuracy thresholds. The simpleness, moreover, illuminates the unknown elegant physics in spin glasses, which is regarded as a complex subject usually.

Since we have considered only frustration to discuss phase transitions, our result seems to support the conjecture on the geometry-induced phase transition $[1,4]$. Nevertheless, our values are different from expected locations from the conjecture $[1,4,18-20,22-25]$. The reason is that we have observed the number of frustrated plaquettes, whereas the entropy of frustration distribution has been considered in the conjecture [1,4]. Specifically, our argument does not take account of the locations of frustrated plaquettes, which is an essential fact in the entropy of frustration distribution. The distribution of frustrated plaquettes might not play an important role in the phase transition at zero temperature. Our result suggests the existence of the geometry-induced phase transition, but it does not support the proposition that the root of the transition at zero temperature is a singularity in the entropy of frustration distribution.

It is necessary to further investigate the models with couplings governed by the Gaussian distribution, for example, the SK model. The examination of the method for the Gaussian distribution is the first step to generalize our method, since random variables with the Gaussian distribution are continuous values and can have a finite mean. We cannot determine yet whether or not our naive generalization of the method developed for the binary couplings to the Gaussian distribution is correct. Let us mention another example of the model with the Gaussian distribution, the Ising spin glass on the hierarchical lattice 1 (Fig. 4). The solution of $v=1$ is $J_{0}=1.623$, and the transition point at zero temperature estimated by the renormalization-group calculation explained in $\S 2$ is $J_{0}=1.216(1)$. The result of our method with frustration is different from the actual transition point as in the SK model. On the analogy with the fact that the solution of the equation $v^{\mathrm{SK}}\left(J_{0}\right)=2 / \pi$ produces the exact transition point in the SK model, we try the similar calculation in the hierarchical lattice 1 . We then obtain the value $J_{0}=1.292$, which is rather close to the actual transition point. This result might be a key to construct the correct generalization of the method to arbitrary distributions.

Since our results have a suggestion about the relationship among frustration, phases, and the replica symmetry, the origin of the agreements might lead to the fruitful structure behind spin glasses in general. In addition, the origin might produce the general rule for the derivation of the accuracy thresholds of topological quantum error-correcting codes. The origin is, however, unclear. Thus, further investigation is necessary. In particular, the lack of the effect from the global structure of lattices and from the nature of spins should be resolved. One of the strategies to examine the influence of the lattice structure is to analyze models on lattices with the bond dilution and contraction [26-28]. It is especially important to clarify the reason why our results are consistent with the transition points of models with the Ising spins. The cases of the Gaussian distribution are also included in future work. 


\section{Acknowledgments}

The author is grateful to H. Nishimori, K. Takahashi, K. Takeda, and M. Ohzeki for valuable discussions. This work was partially supported by the Research Fellowship of the Japan Society for the Promotion of Science.

\section{REFERENCES}

[1] Nishimori, H., Statistical Physics of Spin Glasses and Information Processing: An Introduction, Oxford Univ. Press, Oxford (2001).

[2] Dennis, E., Kitaev, A., Landahl, A., and Preskill, J., “Topological quantum memory,” J. Math. Phys., 43: $4452-4505$ (2002).

[3] Nishimori, H., "Internal energy, specific heat and correlation function of the bond-random Ising model," Prog. Theor. Phys., 66: 1169-1181 (1981).

[4] Nishimori, H., "Geometry-induced phase transition in the $\pm J$ Ising model," J. Phys. Soc. Jpn., 55: 3305-3307 (1986).

[5] Toulouse, G., "Theory of the frustration effect in spin glasses: I," Commun. Phys., 2: 115-119 (1977).

[6] Fujii, K., and Tokunaga, Y., "Error and loss tolerances of surface codes with general lattice structures," Phys. Rev. A, 86: 020303(R) (2012).

[7] Jinuntuya, N., and Poulter, J., "Elementary excitations and the phase transition in the bimodal Ising spin glass model," J. Stat. Mech., 2012: P01010 (2012).

[8] Amoruso, C., and Hartmann, A. K., "Domain-wall energies and magnetization of the two-dimensional random-bond Ising model," Phys. Rev. B, 70: 134425 (2004).

[9] Wang, C., Harrington, J., and Preskill, J., "Confinement-higgs transition in a disordered gauge theory and the accuracy threshold for quantum memory," Ann. Phys., 303: 31-58 (2003).

[10] Achilles, M., Bendisch, J., and Trotha, H. v., "Effect on the groundstate threshold pc by different boundary conditions in 2D $\pm \mathrm{J}$ Ising models," Physica A, 275: 178-196 (2000).

[11] Landahl, A. J., Anderson, J. T., and Rice, P. R., "Fault-tolerant quantum computing with color codes," arXiv:1108.5738 (2011).

[12] Sherrington, D., and Kirkpatrick, S., "Solvable model of a spin-glass," Phys. Rev. Lett., 35: 1792-1796 (1975).

[13] Kirkpatrick, S., "Frustration and ground-state degeneracy in spin glasses," Phys. Rev. B, 16: 4630-4641 (1977).

[14] Vannimenus, J., and Toulouse, G., "Theory of the frustration effect: II. Ising spins on a square lattice," J. Phys. C, 10: L537L542 (1977).

[15] Berker, A. N., and Ostlund, S., "Renormalisation-group calculations of finite systems: Order parameter and specific heat for epitaxial ordering," J. Phys. C, 12: 4961-4975 (1979).

[16] Nobre, F. D., "Phase diagram of the two-dimensional $\pm J$ Ising spin glass," Phys. Rev. E, 64: 046108 (2001).

[17] Bombin, H., and Martin-Delgado, M. A., "Statistical mechanical models and topological color codes," Phys. Rev. A, 77: 042322 (2008).

[18] Katzgraber, H. G., Bombin, H., and Martin-Delgado, M. A., "Error threshold for color codes and random three-body Ising models," Phys. Rev. Lett., 103: 090501 (2009).

[19] Ohzeki, M., "Accuracy thresholds of topological color codes on the hexagonal and square-octagonal lattices," Phys. Rev. E, 80: 011141 (2009).

[20] Katzgraber, H. G., Bombin, H., Andrist, R. S., and Martin-Delgado, M. A., "Topological color codes on Union Jack lattices: A stable implementation of the whole Clifford group," Phys. Rev. A, 81: 012319 (2010).

[21] Toulouse, G., "On the mean field theory of mixed spin glass-ferromagnetic phases," J. Phys. (Paris), Lett., 41: L447-L449 (1980).

[22] Ohzeki, M., "Multicritical points for spin-glass models on hierarchical lattices," Phys. Rev. E, 77: 061116 (2008).

[23] Ohzeki, M., "Locations of multicritical points for spin glasses on regular lattices," Phys. Rev. E, 79: 021129 (2009).

[24] Hasenbusch, H., Toldin, F. P., Pelissetto, A., and Vicari, E., "Multicritical Nishimori point in the phase diagram of the $\pm J$ Ising model on a square lattice," Phys. Rev. E, 77: 051115 (2008).

[25] de Queiroz, S. L. A., "Multicritical point of Ising spin glasses on triangular and honeycomb lattices," Phys. Rev. B, 73: 064410 (2006).

[26] Ohzeki, M., and Fujii, K., "Duality analysis on random planar lattices," Phys. Rev. E, 86: 051121 (2012).

[27] Röthlisberger, B., Wootton, J. R., Heath, R. M., Pachos, J. K., and Loss, D., "Incoherent dynamics in the toric code subject to disorder," Phys. Rev. A, 85: 022313 (2012).

[28] Stace, T. M., and Barrett, S. D., "Error correction and degeneracy in surface codes suffering loss," Phys. Rev. A, 81: 022317 (2010). 